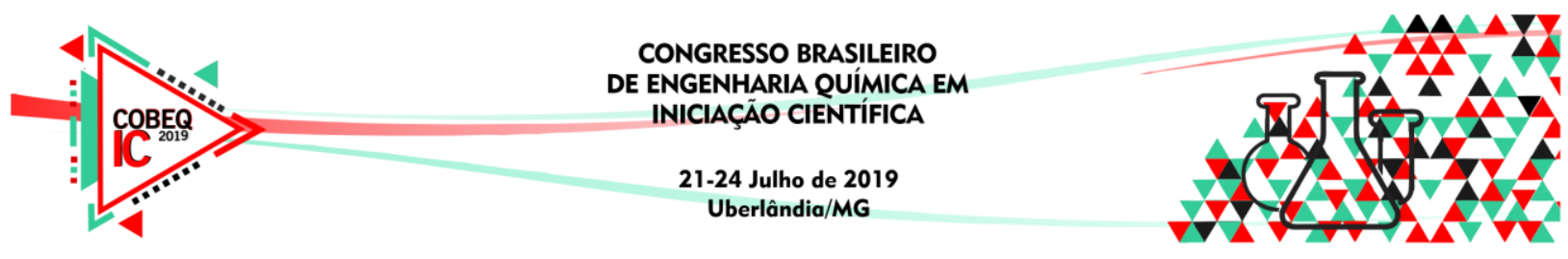

\title{
ANÁLISE E LEVANTAMENTO DAS PRINCIPAIS TÉCNICAS PARA CAPTURA DE $\mathrm{CO}_{2}$
}

\author{
B.W. M. SILVA ${ }^{1}$, J. F. C. FILHO ${ }^{2}$, K.I. REZENDE ${ }^{3}$, M. C. S. SILQUEIRA ${ }^{4}$ e S. N. \\ RABELO $^{5}$ \\ ${ }^{1}$ Universidade de Itaúna, Faculdade de Engenharia Mecânica \\ ${ }^{2}$ Universidade de Itaúna, Faculdade de Engenharia Mecânica \\ ${ }^{3}$ Universidade de Itaúna, Faculdade de Engenharia Mecânica \\ ${ }^{4}$ Universidade de Itaúna, Faculdade de Engenharia Mecânica \\ ${ }^{5}$ Universidade de Itaúna, Faculdade de Engenharia Mecânica e Universidade Federal de \\ Minas Gerais, Departamento de Engenharia Mecânica \\ E-mail para contato: sasanogueirarab@ hotmail.com
}

\begin{abstract}
RESUMO - A crescente emissão do gás carbônico e os impactos causados pelo volume das atuais emissões têm promovido diversos estudos a fim de desenvolver técnicas para capturar esse gás. Dentre as técnicas de captura de dióxido de carbono, destaca-se a absorção, adsorção, tecnologia de membranas e criogenia. $\mathrm{O}$ presente trabalho apresenta um levantamento e uma análise dos avanços atuais dos processos de captura do dióxido de carbono, demonstrando brevemente o mecanismo empregado em cada técnica, bem como alguns dos problemas que cada uma delas enfrenta, e como a comunidade cientifica está se direcionando para resolvê-los. Dentre as técnicas revisadas e analisadas, pode-se destacar a absorção química, uma vez que possui uma maior capacidade de captura de $\mathrm{CO}_{2}$ para uma menor quantidade de energia requerida no processo, comparada com as demais técnicas.
\end{abstract}

\section{INTRODUÇÃO}

Os impactos causados pelo efeito estufa afetam diretamente a economia global. Segundo Stern (2006), o PIB mundial poderá ser afetado por ano em cerca de 5 a $20 \%$ por este fenômeno. A inundação de regiões litorâneas poderá afetar 10 milhões de pessoas por ano se a temperatura terrestre aumentar em $1^{\circ} \mathrm{C}$ anualmente. Apesar do conhecimento em âmbito mundial das alterações climáticas, dados do International Panel of Climate Change (IPCC) indicam que as emissões de carbono continuarão aumentando ano após ano, com consequências que poderão atingir em até 300 milhões de pessoas anualmente em função do aumento do nível do oceano (Stern, 2006).

De acordo com a EPA (Environmental Protection Agency, 2018), 83\% dos gases de efeito estufa nos Estados Unidos em 2016 são lançados à atmosfera devido ao fornecimento de energia, transporte e fins industriais. Os EUA são responsáveis por 15\% da emissão global de carbono, somente atrás da China com 30\% das emissões (IPCC, 2014). Dentre esses gases destaca-se o dióxido de carbono $\left(\mathrm{CO}_{2}\right)$. Na indústria norte americana, o setor de geração de 


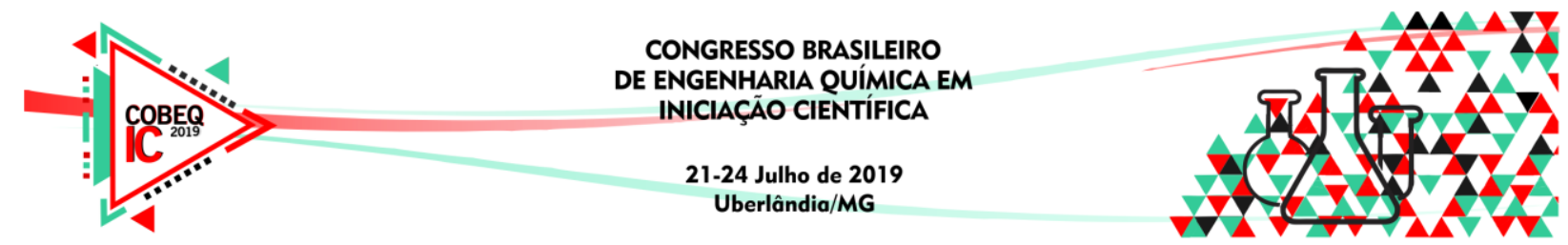

energia elétrica emite $34 \%$ do $\mathrm{CO}_{2}$, perdendo apenas processos industriais que é responsável por $15 \%$ de emissão desse gás.

Várias tecnologias vêm sendo estudadas e aplicadas em escala industrial para separação e recuperação de diversos gases, porém esbarram em problemas como: viabilidade econômica, redução de rendimento total de usinas térmicas, condições específicas de operação que devem ser analisados durante sua implementação, entre outros fatores (Metz et al., 2005).

Dessa forma, nos dias atuais existe uma enorme necessidade de redução da concentração do dióxido de carbono na atmosfera para patamares seguros. O presente trabalho apresenta então um levantamento e uma análise das principais técnicas atuais sobre a captura do $\mathrm{CO}_{2}$, como: a absorção química, a adsorção, a separação por membranas e criogenia.

\section{TÉCNICAS DE CAPTURA DE $\mathrm{CO}_{2}$}

\subsection{Absorção Química}

A captura do $\mathrm{CO}_{2}$ pela absorção química é um processo em que os gases de combustão entram em contato com uma solução de aminas, como exemplo a MEA - Monoetanolamina. Posteriormente, a solução rica em $\mathrm{CO}_{2}$ é aquecida para que o gás absorvido se desprenda da solução. O gás é então comprimido e armazenado para utilização futura (Metz et al., 2005).

Um dos problemas enfrentados por essa técnica é o elevado custo energético demandado na etapa de regeneração, em que é necessário aquecer a solução. Para solucionar este problema, diversos estudos estão voltados para o aperfeiçoamento desta técnica com o uso de aditivos, que quando combinados com as aminas, melhoram as propriedades da solução. Zhang et al. (2017) analisaram o comportamento de soluções com diferentes combinações de MEA (Etanolamina), DEA (Dietanolamina), MDEA (Metildietanolamina), PZ (Piperazina), AMP (2-Amino-2-metil-1-propanol) e concluíram que uma mistura de MEA, MDEA e PZ obteve uma diminuição na energia de regeneração de $15 \%$ a $49 \%$.

Outra linha de pesquisa é o desenvolvimento de catalisadores que imitam anidrases carbônicas. Widger et al. (2017) estudaram catalisadores mímicos de anidrases e concluíram que houve uma redução de pelo menos $20 \%$ na energia envolvida no processo de absorção e regeneração. Também tem se investido em pesquisas sobre o recheio das colunas, a fim de otimizar o fluxo do solvente no interior da coluna e melhorar o contato desses com o gás de combustão. Rahmanian et al (2018) compararam o recheio estruturado BX com recheio randomizado em uma coluna de absorção e regeneração com MEA e concluíram que o recheio estruturado $\mathrm{BX}$ se mostrou mais efetiva pela maior capacidade de absorção do $\mathrm{CO}_{2} \mathrm{e}$ pela menor penalização energética na regeneração, devido a maior área de superfície comparado com o recheio randomizado.

A absorção química é umas das técnicas mais promissoras para captura do $\mathrm{CO}_{2}$, em virtude de sua facilidade de aplicação, eficiência e relativo baixo custo de operação. As novas pesquisas, em suma, objetivam a redução do gasto energético para execução do processo de desabsorção, levando ao desenvolvimento de novas soluções ou novas técnicas para recuperação desse gás após ser absorvido. 


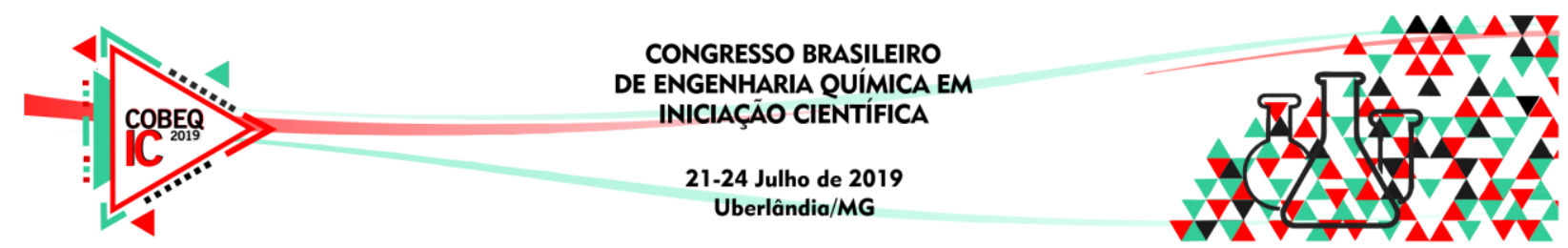

\subsection{Adsorção}

A técnica de captura do $\mathrm{CO}_{2}$ por adsorção baseia-se na interação entre o gás e um sólido, em que as forças de interação podem variar em adsorção física (fisissorção) ou química (quimissorção). Na fisissorção a interação é fraca e do tipo van der Waals. Na quimissorção, a interação é mais forte com a formação de uma monocamada, porém, este é um processo que pode ser lento e irreversível.

Como adsorvatos mais usuais empregados nesse processo, pode-se destacar o carbono ativo, uma vez que possui elevada seletividade em altas pressões e temperaturas ambiente. Além disso, as estruturas dos poros desses componentes podem ser facilmente controladas variando as condições de preparação e ativação (Bezerra, 2010).

Jadhav et al. (2007), estudaram o Zeolite 13X que é um tipo de zeólito que possui uma abertura de poros muito maior comparado com adsorvatos convencionais. Esse adsorvato apresentou uma taxa de transferência muito maior, exibindo uma capacidade de adsorção de $\mathrm{CO}_{2}$ de $28,7 \%$ em peso e uma capacidade de separação em proporção de $3,65 \mathrm{CO}_{2} / \mathrm{N}_{2} \mathrm{em}$ condições de $298 \mathrm{~K}$ e 10 bar. Bezerra (2010) analisou o zeólito modificado 13X com impregnação da MEA para melhorar a capacidade de adsorção de $\mathrm{CO}_{2}$. A eficiência do processo melhorou em 3,5 vezes comparado com o uso desse zeólito não impregnado.

Phan et al., (2010) realizaram uma análise do processo de adsorção para captura de $\mathrm{CO}_{2}$ utilizando os ZIF's (estruturas de imidazolato zeolíticas) que são zeólitos modificados quimicamente) e estruturas metal-orgânicas (MOFs), que são materiais porosos e cristalinos. Como resultado constatou-se que os ZIFs apresentam uma alta afinidade por $\mathrm{CO}_{2}$ a baixas pressões e temperaturas comparado com o MOFs. Além disso, os ZIFs também apresentaram uma maior seletividade do que MOFs para outros gases de combustão relevantes, como o monóxido de carbono.

As principais vantagens do processo adsorção são: a capacidade de operar em diferentes temperaturas, eliminação de problemas de corrosão e menores custos de energia para regeneração. Porém, para que a adsorção de $\mathrm{CO}_{2}$ seja mais eficiente, deve-se melhorar a incorporação de vários grupos aminas, a fim de aumentar a polarização das moléculas, e assim obter um maior rendimento na captura de $\mathrm{CO}_{2}$ (Bezerra, 2010; Lee et al., 2015).

\subsection{Criogenia}

A captura de carbono criogênico é uma tecnologia de pós-combustão que faz com que o $\mathrm{CO}_{2}$ seja dessublimado da corrente do processo por meio da refrigeração e/ou expansão desse gás. A vantagem da separação criogênica é que o $\mathrm{CO}_{2}$ pode ser recuperado com $99 \%$ de pureza. A desvantagem é a alto consumo de energia associada ao resfriamento dos gases por refrigeração. A separação criogênica ocorre pela mudança de fase do $\mathrm{CO}_{2}$, passando-se da fase vapor para líquida ou sólida. A mudança de fase pode ocorrer em temperaturas entre 217 K e 304 K, e a pressões de 630 kPa a 7396 kPa (Osman, Coquelet e Ramjugernath, 2014).

O processo convencional consiste basicamente em condensar o gás em um trocador de calor, de forma eliminar demais impurezas como $\mathrm{CH}_{4}, \mathrm{CO}_{2}, \mathrm{~N}_{2}$ e $\mathrm{O}_{2}$, além de traços de $\mathrm{Hg}$, $\mathrm{SO}_{2}$ e $\mathrm{HCl}$. O dióxido de carbono é então comprimido e enviado para outro trocador de calor 


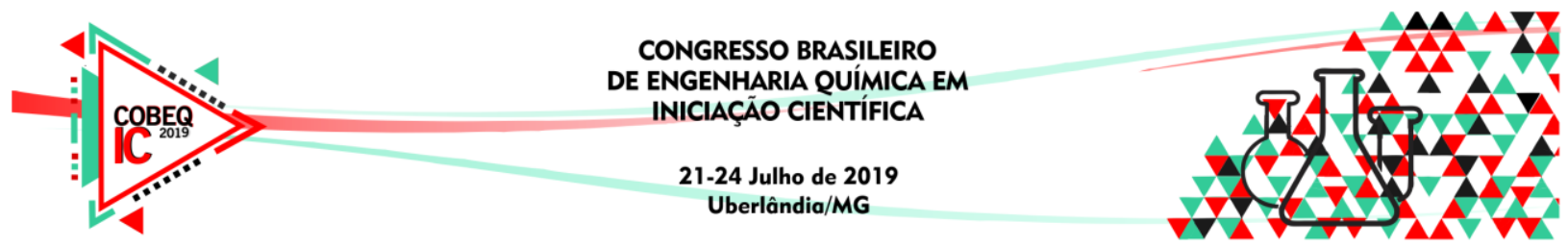

para que a temperatura atinja um ponto um pouco abaixo da temperatura de solidificação do $\mathrm{CO}_{2}$, a qual varia de acordo com a pressão do sistema. Posteriormente mecanismos separam os elementos restantes com base em sua volatilidade (Osman et al., 2014).

Song et al, 2017 propôs um processo que se inicia-se com bombeamento dos gases de combustão abaixo de $50^{\circ} \mathrm{C}$ e compressão entre $101 \mathrm{kPa}$ a $202 \mathrm{kPa}$. Durante o processo, a água presente nos gases condensa, e assim este é enviado a seco $\left(10^{\circ} \mathrm{C}\right)$ para a torre principal de congelamento. $\mathrm{O}$ gás residual é separado e exaurido, e assim o $\mathrm{CO}_{2}$ na mistura é capturado na fase sólida a $140^{\circ} \mathrm{C}$. Após esse processo, o $\mathrm{CO}_{2}$ é comprimido até a pressão de transporte do gasoduto $(103 \mathrm{kPa})$. Os resultados obtidos pelos autores mostraram que o uso de energia para recuperação do $\mathrm{CO}_{2}$ diminui consideravelmente ao longo de um ano, em virtude da utilização de uma usina a base de carvão.

Jensen et al. (2015) investigaram a eficiência de captura de $\mathrm{CO}_{2}$ por criogenia utilizando-se uma usina elétrica movida a carvão em conjunto com um circuito de resfriamento externo. A eficiência do processo alcançou $90 \%$ de captura de $\mathrm{CO}_{2}$, além de outros gases nocivos. Sayre et al. (2017) testaram misturas de carvão e biomassa, como o carvão wyoming black thunder, betuminoso e sub-betuminoso, gás natural, entre outros. Para a maioria das misturas analisadas, observou-se que a taxa de captura de $\mathrm{CO}_{2}$ foi maior que $98 \%$, comprovando-se a eficiência desta técnica, apesar dos custos energéticos.

\subsection{Membranas}

Um dos métodos mais recentes para captura do dióxido de carbono e outros gases consiste no uso de membranas. Por não ser uma técnica muito bem desenvolvida ainda, não se sabe com exatidão como ocorre o processo da captura do $\mathrm{CO}_{2}$ (Belaissaoui e Favre, 2013).

O princípio de operação dessa técnica baseia-se na obtenção de uma barreira física, pela qual o gás rico em $\mathrm{CO}_{2}$ é forçado a passar. Espera-se que o dióxido de carbono seja difundido nessa superfície, enquanto que os demais gases consigam atravessá-la. Tal sistema possui como força motriz a diferença de pressão parcial na entrada e saída da membrana (Reis, 2013). Assim, faz-se necessário que a membrana seja permeável, isto é, o $\mathrm{CO}_{2}$ deve interagir com o material da membrana para ser capaz de se difundir. No entanto, essa membrana também deve ser seletiva, permitindo que apenas a substância desejada seja filtrada.

Destaca-se o óxido de cálcio $(\mathrm{CaO})$ como material para fabricação dessas membranas. Devido a sua estrutura cristalina e de elevada porosidade, o $\mathrm{CaO}$ possui grandes expectativas para ser um material base para as membranas. Estudos sugerem, que, em membranas produzidas a partir de $\mathrm{CaO}$ oriundos de cascas de ovos de galinha usado na reação de síntese de $\mathrm{CaCO}_{3}$, alcançam um aumento significativo na captura de $\mathrm{CO}_{2}$. Tal remoção já chegou a níveis quase similares aos de absorção em membrana fabricadas a partir de $\mathrm{CaO}$ comercial (Vieira et al., 2017). Deve-se ressaltar que além da captura de $\mathrm{CO}_{2}$ por $\mathrm{CaO}$, tem-se a redução da geração de resíduos, uma vez que ovos fazem parte da dieta de grande parte da população mundial. (Mohammadi, Lahijani e Mohamed, 2014).

Atualmente, os principais problemas enfrentados na aplicação da técnica de captura de $\mathrm{CO}_{2}$ por meio de membranas estão relacionados com a seletividade e permeabilidade do 


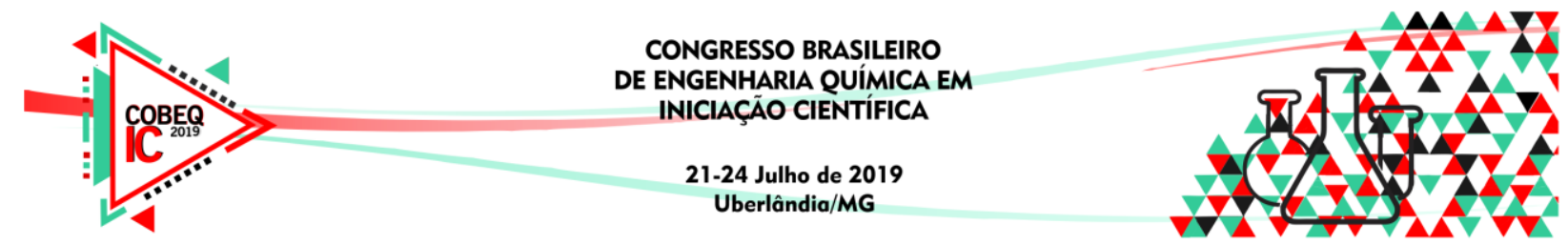

material, além da energia de regeneração gasta no processo (Tachy, 2016). Nesse contexto, observa-se a importância do estudo dos materiais para fabricação das membranas (Bui et al., 2018). Apesar disso, pode-se dizer que a captura de dióxido de carbono por meio de membrana é um método recente e muito promissor.

\section{CONCLUSÃO}

Algumas técnicas como a tecnologia de membranas e adsorção carecem de mais estudos, tanto para testes em escala laboratorial, bem como em escala industrial, devido à problemas como a necessidade de regeneração no caso da adsorção e as baixas taxas de captura de ambas as técnicas apresentadas. Já a absorção química, ainda pode ser necessária uma análise cuidadosa da viabilidade energética do processo devido à energia despendida na etapa de regeneração. Mesmo assim, esta técnica é a que se mostra estar melhor desenvolvida, além de apresentar melhor relação entre carbono absorvido e energia requerida ao processo. Conclui-se que os esforços das pesquisas atuais estão voltados para aumentar a eficiência das técnicas de captura de $\mathrm{CO}_{2}$, reduzindo o gasto energético, principalmente na fase de regeneração. Desse modo, em trabalhos futuros, espera-se desenvolver em escala laboratorial o processo de absorção por aminas primárias e secundárias, uma vez que essa foi a técnica que apresentou o melhor custo benefício e simplicidade de aplicação para captura do $\mathrm{CO}_{2}$. Diferentes soluções de aminas deverão ser analisadas a fim de se determinar a melhor composição para o processo de absorção, bem como melhorias de layout da coluna de absorção serão propostas para melhorar o aproveitamento energético da aplicação desta técnica.

\section{REFERÊNCIAS}

BELAISSAOUI, B.; FAVRE, E. Membrane Separation Processes for Post-Combustion Carbon Dioxide Capture: State of the Art and Critical Overview. Oil \& Gas Science And Technology, v. 69, n. 6, p.1005-1020, 2013.

BEZERRA, D. P. Captura de $\mathrm{CO}_{2}$ - estudo do equilíbrio de adsorção por técnica gravimétrica com adsorventes comerciais e modificados. Dissertação (Mestrado em Engenharia Química) - Centro de Tecnologia, Universidade Federal do Ceará, Fortaleza, 2010.

BUI, M. et al. Carbon capture and storage (CCS): the way forward. Energy \& Environmental Science, v.11, n.5, p.1062-1176, 2018.

EPA. Inventory of U.S. Greenhouse Gas Emissions and Sinks: 1990-2016. 2018. Disponível em: $<$ https://www.epa.gov/sites/production/files/201801/documents/2018_complete_report.pdf>. Acesso em: 06 mar. 2019.

JADHAV, P. D. et al. Monoethanol amine modified zeolite $13 \mathrm{X}$ for $\mathrm{CO}_{2}$ adsorption at different temperatures. Energy \& Fuels, v. 21, n.6, p.3555-3559, 2007.

IPCC. Climate Change 2014 Mitigation of Climate Change. 2014. Disponível em: <https://www.ipcc.ch/site/assets/uploads/2018/02/ipcc_wg3_ar5_frontmatter.pdf>. Acesso em: 9 fev. 2019. 


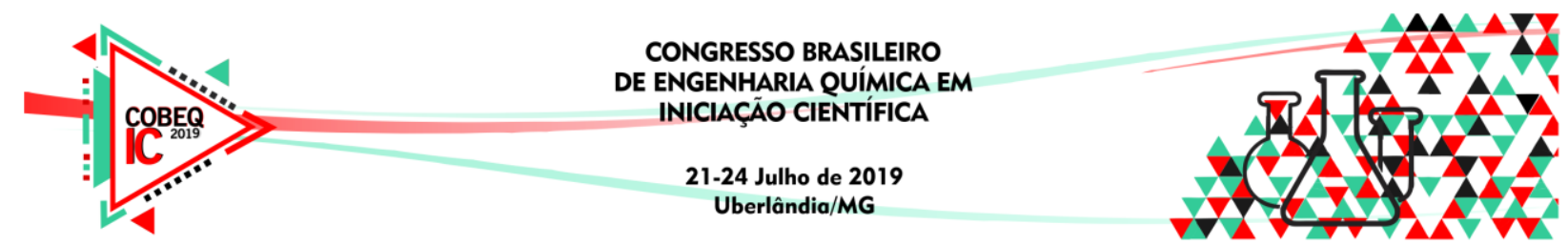

LEE, S.; PARK, S. A review on solid adsorbents for carbon dioxide capture. Journal of Industrial And Engineering Chemistry, v. 23, p.1-11, 2015.

METZ, B. et al. IPCC Special Report on Carbon Dioxide Capture and Storage. Cambridge, United Kingdom and New York, USA: Cambridge University Press, 2005. 442 p.

MOHAMMADI, M.; LAHIJANI, P.; MOHAMED, A. R. Refractory dopant-incorporated $\mathrm{CaO}$ from waste eggshell as sustainable sorbent for $\mathrm{CO}_{2}$ capture: Experimental and kinetic studies. Chemical Engineering Journal, v. 243, p.455-464, 2014.

OSMAN, K.; COQUELET, C.; RAMJUGERNATH, D. Review of carbon dioxide capture and storage with relevance to the South African power sector. Pretoria, v.110, n. 5-6, p. 01-12, 2014.

PHAN, Anh et al. Synthesis, structure, and carbon dioxide capture properties of zeolitic imidazolate frameworks. Acc. Chem. Res, v. 43, n. 1, p. 58-67, 2010.

RAHMANIAN, N., REHAN, M., SUMANI, A., NIZAMI, A. S. Effect of Packing Structure on $\mathrm{CO}_{2}$ Capturing Process. Chemical Engineering Transactions, v.70, p.1891-1896, 2018 .

REIS, C. V. Desenvolvimento de tecnologias de captura e armazenamento de $\mathrm{CO}_{2}$. Dissertação (Mestrado) - Curso de Ciências da Natureza, Universidade Nova de Lisboa, Lisboa, 2013.

STERN, N. Review report on the economics of climate change. 2006. Disponível:em:http://mudancasclimaticas.cptec.inpe.br/ rmclima/pdfs/destaques/sternre view_report_complete.pdf. Acesso em: 26 Jun 2018.

TACHY, D. E. Avaliação técnica e econômica da captura de $\mathrm{CO}_{2}$ por membrana, absorção e tecnologias híbridas. Dissertação (Mestrado) - Curso de Engenharia Industrial, Programa de Pós-Graduação em Engenharia Industrial - Pei, Universidade Federal da Bahia, Salvador, 2016

VIEIRA, L. A. F. et al. Obtenção de Óxido de Cálcio a partir da Casca de Ovo de Galinha. The Journal of Engineering and Exact Sciences, v.3, n.8, p.1159-1166, 2017.

WIDGER, L. R. et al. Enhancements in mass transfer for carbon capture solvents part I: Homogeneous catalyst. International Journal of Greenhouse Gas Control, v.63, p.249259, 2017.

ZHANG, R. et al. Analysis of the reduction of energy cost by using MEA-MDEA-PZ solvent for post-combustion carbon dioxide capture (PCC). Applied Energy, v.205, p.1002$1011,2017$. 